\title{
Ring finger protein 43 associates with gastric cancer progression and attenuates the stemness of gastric cancer stem-like cells via the Wnt- $\beta$ /catenin signaling pathway
}

Yunhe Gao ${ }^{1+}$, Aizhen $\mathrm{Cai}^{1+}$, Hongqing $\mathrm{Xi}^{1+}$, Jiyang $\mathrm{Li}^{1+}$, Wei X ${ }^{2}$, Yanmei Zhang ${ }^{2}$, Kecheng Zhang ${ }^{1}$, Jianxin Cui ${ }^{1}$, Xiaosong $\mathrm{Wu}^{1}$, Bo Wei ${ }^{1}$ and Lin Chen ${ }^{1 *}$

\begin{abstract}
Background: Ring finger protein 43 (RNF43) is a member of the transmembrane E3 ubiquitin ligase family that was originally found in stem cells and plays important roles in tumor formation and progression. Our previous study indicated that RNF43 might be a tumor suppressor protein in gastric cancer. Given its antagonistic relationship with leucine-rich repeat-containing G-protein-coupled receptor 5 (Lgr5), one of the gastric cancer stem cell markers, investigation of the potential role of RNF43 in gastric stem cancer cells is necessary.

Methods: Immunohistochemistry staining, western blot analysis, and quantitative reverse transcription polymerase chain reaction were used to determine the mRNA and protein expression level of RNF43 and other Wnt pathway factors. Gastric cancer stem-like cells were obtained from gastric cancer tumor and cell lines by tumorsphere culture. The adeno-associated virus system was used to upregulate RNF43 expression in cancer cells. Functional experiments including tumorsphere formation, chemotherapy resistance, surface marker detection, and tumor xenograft assay were performed to measure stem-like properties in gastric cancer stem-like cells after RNF43 overexpression.

Results: RNF43 loss was significantly associated with TNM stage, distant metastasis, and Lauren classification, and predicted worse prognosis in gastric cancer patients. RNF43 expression was even lower in tumorspheres derived from tumor tissues or cell lines compared with adherent cancer cells and normal gastric cells. Overexpression of RNF43 in gastric cancer cells impaired their stem-like properties, including sphere formation ability, chemoresistance in vitro, and tumorigenicity in vivo. Moreover, Wnt pathway-related proteins were decreased in RNF43-overexpressing cells, while Wnt pathway activators could reverse the trend to some extent.
\end{abstract}

Conclusions: Our findings indicated that RNF43 might not only participate in gastric cancer progression, but also attenuate the stemness of gastric cancer stem-like cells through the Wnt/ß-catenin pathway.

Keywords: Ring finger protein 43, Gastric cancer, Stem cells, Stem-like property

\footnotetext{
* Correspondence: chenlinbj@sina.com

${ }^{\dagger}$ Equal contributors

'Department of General Surgery, Chinese PLA General Hospital, Beijing

100853, China

Full list of author information is available at the end of the article
} 


\section{Background}

Gastric cancer (GC) is the fourth most common malignancy and the second leading cause of cancerrelated death worldwide [1]. Despite breakthroughs in basic research and clinical management, the prognosis of patients with GC is still rather poor, with an average 5-year survival rate of around 30\% [2]. Many GC patients die from tumor metastasis, relapse, and failure of chemotherapy.

Cancer stem cells (CSCs) are defined as a subpopulation of cells that possess self-renewal and differentiation ability [3]. Gastric CSCs have been isolated and identified from GC cell lines or tumor tissues using a serial of methods and surface markers [4-6]. Although increasing evidence has emerged to support the existence of gastric CSCs, less is known about their underlying regulatory mechanism [7]. Better understanding of the biology and mechanisms of CSCs might help improve the understanding of carcinogenesis, progression, and chemoresistance of GC.

E3 ubiquitin ligases are a large family of proteins that regulate the structure and activity of many target proteins [8]. Ring finger protein 43 (RNF43) is an E3 ubiquitin ligase originally found in stem cells [8] and is mutated in several kinds of carcinomas. Early findings described RNF43 as an oncoprotein highly expressed in colorectal tumors [9], whereas more recent research has supported its function as a tumor suppressor protein in pancreatic cancer [10], ovary cancer [11], hepatocellular cancer [12], and GC [13].

Recently, RNF43 was found to be commonly mutated in GC through whole-genome sequencing analysis [14]. RNF43 was also reported as a negative regulator of the Wnt signaling pathway, which is instrumental for development and tumor carcinogenesis. RNF43 inhibits Wnt signaling by directly ubiquitinating frizzled receptors and its downstream pathway [15]. RNF43 and its homolog, ZNRF3, are antagonized by binding of leucinerich repeat-containing G-protein-coupled receptor 5 (Lgr5) and its ligand R-spondin. Lgr5 is an important Wnt upstream receptor [16] and considered one of the stem cell markers in the gastrointestinal tract and malignancies [17-19].

In this study, we examined the precise role of RNF43 in gastric CSCs and the underlying mechanism.

\section{Methods}

\section{Clinical samples and cell lines}

A total of 93 paired specimens (tumor and adjacent nontumor tissues at least $5 \mathrm{~cm}$ away from tumor) were obtained from GC patients who underwent surgery from 2010 to 2013 at the Department of General Surgery, Chinese PLA General Hospital (Beijing, China). This study was approved by the Institutional
Review Board of the Chinese PLA General Hospital and all patients provided written informed consent. Clinicopathological information was extracted from patients' medical records.

\section{Isolation of primary gastric tumor cells}

Fresh human GC tissues were obtained immediately after resection from patients. All samples were transported to the laboratory on ice within $30 \mathrm{~min}$ and immediately disaggregated mechanically by scissors into 1$\mathrm{mm}$ pieces. Tumor pieces were then digested by intermittent pipetting in phosphate buffer saline (PBS) solution with $1 \mathrm{mg} / \mathrm{ml}$ collagenase I and $1 \mathrm{mg} / \mathrm{ml}$ collagenase IV (Life Technologies, Waltham, MA, USA) at $37{ }^{\circ} \mathrm{C}$ for $1 \mathrm{~h}$. Tumor digestion was terminated with DMEM/F12 medium containing 20\% fetal bovine serum (FBS; Life Technologies).

\section{Tumorsphere culture and formation}

Digested cells from primary gastric tumors or GC cell lines were cultured to form spheres in ultralowattachment six-well and 24-well plates (Corning, NY, USA) at a density of 10,000 cells $/ \mathrm{ml}$ in DMEM/F12 medium with $2 \%$ B27 (Invitrogen, CA, USA), EGF (20 ng/ml; Invitrogen), bFGF (10 ng/ml; Invitrogen), LIF (10 ng/ml; Peprotech, Hartford, CT, USA), and Hepes (Invitrogen). GC cells were cultured in DMEM/ F12 medium with or without the addition of Wnt5a (200 ng/ml; R\&D System), DKK-1 (100 ng/ml; R\&D System), and R-spondin 1 (200 ng/ml; R\&D System) to form spheres. Spheres with diameter $>100 \mu \mathrm{m}$ were counted 7 days after being planted.

\section{RNA isolation and quantitative real-time PCR}

Total RNA was extracted from tissue samples and cell lines using TRIzol reagent according to the manufacturer's instructions (Invitrogen). First-strand cDNA was synthesized using HiScriptQ RT SuperMix for qPCR (Vazyme, Nanjing, China). Quantitative real-time PCR was performed using AceQ SYBR Master Mix (Vazyme, Nanjing, China) on a 7900 HT system. The PCR primers used to amplify target genes were as follows: RNF43, forward 5'-CAAATTCACAGCCAGTGTGG-3' and reverse $5^{\prime}$-GTCCTTTCCTTTCCCAGGAG-3'; $\beta$-actin, forward 5'-GCTCGTCGTCGACAACGGCTC-3' and reverse 5'-CAAACATGATCTGGGTCATCTTCTC-3'; and Sox-2, forward 5'-GGATGGTTGTCTATTAACTT$3^{\prime}$ and reverse 5'-TCAAACTTCTCTCССТTT-3'. $\beta$ actin was used as the reference gene. The $\mathrm{Ct}$ values of the samples were calculated, and the relative levels of mRNA were analyzed by the $2^{-\Delta \Delta C T}$ method. Each sample was analyzed in triplicate to minimize the stochastic error. 


\section{Construction of adenovirus encoding RNF43}

The RNF43 cDNA and the green fluorescence protein gene (Ad-GFP) cloned by PCR were inserted into the pDC315-EGFP vector (purchased from Hanbio Co. Ltd, Shanghai, China) under the control of the mouse cytomegalovirus (CMV) promoter. The pDC315-X and pBHGlox E1,3 Cre plasmids were cotransfected into HEK293 cells to generate the recombinant adenoviruses. Ad-RNF43 and Ad-GFP were propagated in HEK293 cells. The propagated recombinant adenoviruses were purified and the titers of virus were concentrated up to $1 \times 10^{10}$ plaque formation unit (PFU)/ $\mathrm{ml}$. The primers used in PCR for RNF43 cDNA generation were as follows: RNF43, forward $5^{\prime}$-CAAC GAATTCATGAGTGGTGGCCACCAGCTGC-3' and reverse 5' -ATTAGCGGCCGCTTACACAGCCTGTT CACACAGCTCC-3'.

\section{Immunohistochemistry and evaluation}

Sections (5 $\mu \mathrm{m}$ thick) were cut from paraffin-fixed, paraffin-embedded tissues. The slides were dewaxed in xylene and rehydrated. The slides were heated in $0.01 \mathrm{~mol} / \mathrm{L}$ citrate buffer $(\mathrm{pH} 6.0)$ in a microwave oven for $4 \mathrm{~min}$ at $100{ }^{\circ} \mathrm{C}$ for antigen retrieval. The slides were washed with PBS and blocked with 10\% goat serum. Sections were treated with primary polyclonal rabbit antibody against RNF43 (ab129401, 1:200; Abcam, MA, USA), $\beta$-catenin (\#8480, 1:200; Cell Signaling Technology, Danvers, MA, USA), and Sox-2 (\#14962S, 1:100; Cell Signaling Technology) and incubated overnight at $4{ }^{\circ} \mathrm{C}$. After washing with PBS, the sections were incubated for $30 \mathrm{~min}$ with biotinylated secondary antibody (ZSGB-BIO, Beijing, China) and peroxidase reactivity was visualized using a 3,30-diaminobenzidine (DAB) substrate kit (ZSGB-BIO). The slides were then counterstained with hematoxylin. The primary antibody was replaced with PBS as a negative control. The staining score evaluation was performed by two independent pathologists. Scores for intensity was as follows: 0, no staining; $1+$, weak staining; $2+$, moderate staining; and $3+$, intense staining. The percentage score was as follows: 0 , no staining of any cells; $1+$, positive staining in up to $30 \%$ cells; $2+$, positive staining in $31-60 \%$ cells; and $3+$, positive staining in $61-100 \%$ cells. The final score was determined as the combination of these two scores, for which final score $\leq 2$ was considered negative and score $\geq 3$ was considered positive.

\section{Western blot analysis}

Quantification of protein lysates was measured with a protein BCA assay Kit (Bio-Rad). Protein samples were separated by SDS-PAGE and transferred to a nitrocellulose membrane. The membranes were blocked with $5 \%$ nonfat milk in TBST $(50 \mathrm{mmol} / \mathrm{L}$ Tris- $\mathrm{HCl}(\mathrm{pH} 7.6)$,
$150 \mathrm{mmol} / \mathrm{L} \mathrm{NaCl}, 0.3 \%$ Tween 20) for $1 \mathrm{~h}$ at room temperature and then incubated in blocking buffer at $4{ }^{\circ} \mathrm{C}$ overnight with primary antibody: anti-RNF43 (SAB2102033, 1:200; Sigma-Aldrich, St. Louis, MO, USA), anti- $\beta$-catenin (\#8480S, 1:1000), anti-RNF75 (\#11922, 1:1000), anti-Cul4A (\#2699, 1:1000), anti-Cmyc (\#5605, 1:2000), anti-TCF4 (\#2565, 1:2000), and anti- $\beta$-actin (\#3700, 1:5000; all Cell Signaling Technology). After washing with TBST, the membranes were incubated with horseradish peroxidase-coupled goat anti-rabbit/ mouse secondary antibody (1:10,000; Easybio, Beijing, China) for $2 \mathrm{~h}$ at room temperature. Detection was performed with enhanced chemiluminescence according to the manufacturer's instructions (ECL kit, Life Technologies).

\section{Flow cytometry analysis}

Single cells digested by accutase (Life Technologies) from spheres in HBSS buffer (Life Technologies) were stained with stem cell markers for $20 \mathrm{~min}$ at $4{ }^{\circ} \mathrm{C}$. Cells were then washed with HBSS buffer for analysis on BD Accuri C6 (BD Bioscience, MD, USA). The stem cell markers include $\mathrm{PE}-\mathrm{CY} 7$ conjugated anti-human CD44 (\#560533, BD Bioscience) and FITC-conjugated antihuman CD54 (\#353107, Biolegend, San Diego, CA, USA). DAPI (Sigma-Aldrich) at a final concentration of $1 \mu \mathrm{g} / \mathrm{ml}$ was used to separate the live and dead cells. Raw data were analyzed with FlowJo software (Version 10.0.7; FlowJo, Ashland, OR, USA).

\section{Proliferation and chemoresistance assays}

The Cell Counting Kit-8 (CCK-8; Dojindo, Kumamoto, Japan) was used to measure cell viability after RNF43 overexpression (OE). The cells were plated in DMEM (Invitrogen) at a density of 5000 cells per well in 96-well plates. CCK- 8 assays were performed $0,24,48$, and $72 \mathrm{~h}$ after infection. CCK- 8 reagent $(10 \mu \mathrm{l})$ was added to each well and the cells were incubated for $1 \mathrm{~h}$ at $37^{\circ} \mathrm{C}$. The $\mathrm{OE}$ and control cells were treated with chemotherapy reagent including $2.5 \mu \mathrm{g} / \mathrm{ml} \mathrm{5-fluorouracil} \mathrm{(5-Fu)} \mathrm{(Sigma-}$ Aldrich), $0.25 \mu \mathrm{g} / \mathrm{ml}$ oxaliplatin (Sigma-Aldrich), and DMSO as control. The medium for each well was replaced by normal DMEM after $72 \mathrm{~h}$ of treatment. Viable cell counts were estimated by measuring the optical density at $450 \mu \mathrm{m}$.

\section{Cell apoptosis assay}

Cell apoptosis was measured $48 \mathrm{~h}$ after treating with 5$\mathrm{Fu}(1 \mu \mathrm{g} / \mathrm{ml})$ or oxaliplatin $(2.5 \mu \mathrm{g} / \mathrm{ml})$ using flow cytometry with the APC-Annexin V Apoptosis Detection Kit (Biolegend) according to the manufacturer's instructions. Briefly, HGC-27 and NCI-87 cells were harvested after treatment, then washed with HBSS, resuspended in $200 \mu \mathrm{l}$ binding buffer, and incubated with $5 \mu \mathrm{l}$ Annexin 
$\mathrm{V}$-APC and $10 \mu \mathrm{l}$ PI for $20 \mathrm{~min}$ at room temperature. Subsequently, the number of stained cells was assessed with a flow cytometer (Accuri C6; BD Bioscience).

\section{In-vivo subcutaneous xenograft assay}

Suspensions of RNF43-overexpressing or control cells $\left(5 \times 10^{6}\right.$ cells) mixed with matrigel (Corning) at a 1:1 ratio $(200 \mu \mathrm{l}$ per mouse) were injected into the rear flank of 4-week-old male NOD;Scid;ll2 $\mathrm{rg}^{-/-}$(NSG) mice to establish the xeno-transplant tumors. Tumor sizes were measured using a vernier caliper every 2 days and the tumor volume was calculated with the formula:

$$
V=0.5 \times \text { length } \times \text { width }^{2} .
$$

Once xenograft tumors reached about $1000 \mathrm{~mm}^{3}$ after 6-8 weeks, mice were sacrificed and tumors were dissected. Tumors were immediately fixed by formalin for $24 \mathrm{~h}$ and then embedded in paraffin. Immunohistochemical staining of RNF43 and $\beta$-catenin was performed in xenograft tumor slides according to the standard procedure already described.

\section{Statistical analysis}

SPSS version 19.0 (SPSS Inc., Chicago, IL, USA) was applied for all statistical analysis. The results of experiments compromising two groups were analyzed using a two-tailed Student's $t$ test. Pearson's $\chi^{2}$ test was applied to assess the various clinicopathological characteristics as a function of RNF43 expression determined by immunohistochemical analysis. Cumulative survival curves were drawn using the Kaplan-Meier method. The difference between the curves was analyzed using the logrank test. $P<0.05$ was considered statistically significant.

\section{Results}

RNF43 expression in GC

We first examined RNF43 protein expression in GC tumor tissues and normal tissues by immunohistochemistry. We detected a trend for decreased RNF43 expression in cancer tissues compared with the corresponding normal tissues $(P<0.01$; Fig. 1a). RNF43 expression was observed in the cytoplasm and nucleus of normal epithelial cells, and the positive expression was more often detected in the lower part of the gastric epithelial layer (Fig. 1a).

Furthermore, we also detected RNF43 expression in other cancer types and their adjacent normal tissues using the same IHC staining method. The results showed that the expression of RNF43 was decreased compared with adjacent normal tissues in colon cancer, while in lung and ovarian cancer the expression in cancer tissues and normal tissues did not exhibit a significant difference (Additional file 1: Figure S1).

\section{Correlation of RNF43 expression with clinical variables}

The association of RNF43 protein expression with the major clinicopathological features of $93 \mathrm{GC}$ cases is presented in Table 1. Decreased RNF43 expression was found to be significantly associated with distant metastasis $(P=0.03)$, TNM stage $(P=0.033)$, and Lauren classification $(P=0.01)$. Moreover, lack of RNF43 expression could predict poorer overall survival of GC patients (log-rank test, $P=0.008$; Fig. 1c). RNF43-negative patients exhibited a shorter survival time (median months, mean $21.7 \pm 9.2$ months) than RNF43-positive patients (mean $28.9 \pm 6.8$ months).

\section{RNF43 expression in GC stem-like cells}

Several different approaches have typically been adapted for the enrichment of CSCs $[20,21]$. We used the 'spheroid colony formation' method to identify gastric cancer stem-like cells (GCSLCs) in this study, which involves culturing potential CSCs with serum-free medium containing EGF and bFGF. The growth of spherical colonies would be considered indicative of stem-like cells with self-renewal ability. Spheroid cells (SCs) were successfully obtained from HGC-27 and NCI-87 GC cell lines while AGS GC cells could not form spheres (Additional file 2: Figure S2). All tumorspheres were maintained in culture for at least 14 days and passed three times to assure self-renewal ability. Stemness associated protein Sox-2 (SRY-box 2) [22] was also measured (Fig. 2a) to confirm sphere stem-like properties. Together these results indicated that the SCs could be considered GCSLCs.

The expression of RNF43 in GC cells and GCSLCs from HGC-27 and NCI-87 cell lines was next examined by western blot assay. Compared with the adherent cells (ACs), SCs exhibited decreased expression of RNF43, and the NCI-87 SCs even showed negative expression of RNF43 (Fig. 2a). Moreover, two other members of E3 ubiquitin ligases, RNF75 and Cul4a, were measured by western blot. Although expression of RNF75 and Cul4a in GC cells (HGC-27 and NCI-87) was different from that in GES-1 cells, the expression of these two E3 ubiquitin ligases between ACs and SCs showed no significant difference (Fig. 2a). We also performed qRT-PCR to analyze RNF43 mRNA expression in GCSLCs and ACs. The mean fold-change of RNF43 was significantly lower in GCSLCs than ACs, which was consistent with the protein levels (Fig. 2b).

To further confirm our findings, SCs were also obtained successfully from two GC patient tumor samples, HSC034 and HSC035, using the method already described. These clinical tumorspheres were maintained in culture for at least 2 months and passed three times to assure self-renewal ability (Fig. 2c). Western blot assay and qRT-PCR demonstrated that the expression of 


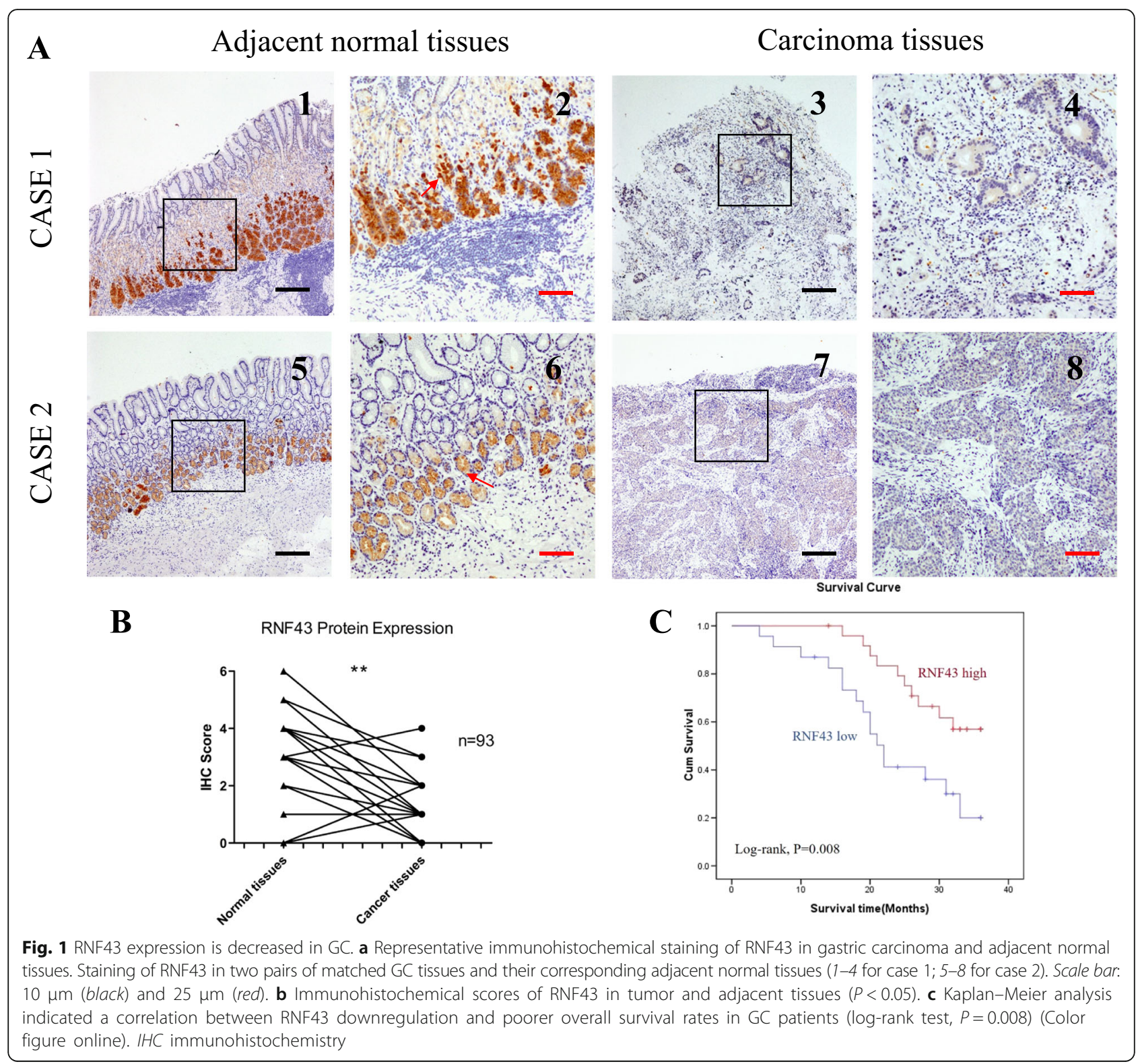

RNF43 was lost in clinical tumorspheres compared with corresponding tumor tissues and adjacent normal tissues (Fig. 2d, e).

RNF43 OE attenuates the stem-like properties of GSCLCs Giving the finding that RNF43 expression was decreased in GC cell lines and GCSLCs, we next constructed a recombinant adenovirus carrying the RNF43 gene (AdRNF43). HGC-27 and NCI-87 cells were infected with Ad-RNF43 and the negative control Ad-EGFP adenovirus (Additional file 3: Figure S3) and the OE efficiency was confirmed by western blot assay. We examined the cell viability of RNF43 OE cells and control groups using CCK- 8 assays and found that RNF43 OE significantly suppressed cell proliferation compared with control groups in a time-dependent manner $(P<0.05$; Fig. $3 \mathrm{~b})$.

One of the important features of CSCs is resistance to chemotherapy, which could be attributed to the heterogeneity of cancer cells differentiated from CSCs. We examined the chemoresistance ability of RNF43 OE and control groups to commonly used chemotherapy drugs in $\mathrm{GC}$, such as 5-Fu and oxaliplatin, with CCK-8 assays. RNF43 OE cells from HGC-27 and N87 cells showed significantly lower sensitivity with both chemotherapy drugs than control cells $(P<0.01)$ (Fig. 3a). Under certain concentrations of chemotherapy, HGC-27 OE and NCI-87 OE cell proliferation was significantly suppressed compared with control cells (Additional file 4: Figure S4). To explore the reason for RNF43 OE cells' 
Table 1 Clinico-pathological variables and the expression of RNF43 in total gastric cancer patients

\begin{tabular}{|c|c|c|c|}
\hline Characteristic & RNF43-positive & RNF43-negative & $P$ value \\
\hline \multicolumn{4}{|l|}{ Age (years) } \\
\hline$<50$ & 14 & 19 & \multirow[t]{2}{*}{0.694} \\
\hline$>50$ & 28 & 32 & \\
\hline \multicolumn{4}{|l|}{ Gender } \\
\hline Male & 20 & 35 & \multirow[t]{2}{*}{0.761} \\
\hline Female & 15 & 23 & \\
\hline \multicolumn{4}{|c|}{ Depth of invasion } \\
\hline $\mathrm{T} 1$ & 1 & 3 & \multirow[t]{4}{*}{0.966} \\
\hline $\mathrm{T} 2$ & 10 & 22 & \\
\hline T3 & 13 & 24 & \\
\hline T4 & 7 & 13 & \\
\hline \multicolumn{4}{|c|}{ Lymph node metastasis } \\
\hline No & 12 & 8 & \multirow[t]{4}{*}{0.075} \\
\hline $\mathrm{N} 1$ & 13 & 31 & \\
\hline $\mathrm{N} 2$ & 5 & 15 & \\
\hline N3 & 3 & 6 & \\
\hline \multicolumn{4}{|c|}{ Distant metastasis } \\
\hline Negative & 35 & 37 & \multirow[t]{2}{*}{$0.030^{C *}$} \\
\hline Positive & 4 & 17 & \\
\hline \multicolumn{4}{|l|}{ pTNM stage } \\
\hline । & 9 & 10 & \multirow[t]{4}{*}{$0.033^{*}$} \\
\hline$\|$ & 12 & 14 & \\
\hline III & 4 & 23 & \\
\hline IV & 5 & 16 & \\
\hline \multicolumn{4}{|c|}{ Lauren classification } \\
\hline Intestinal & 12 & 48 & \multirow[t]{2}{*}{$0.01^{*}$} \\
\hline Diffuse & 15 & 18 & \\
\hline
\end{tabular}

$C$ continuity correction

*Statistically significant $(P<0.05)$

impaired chemotherapy resistant ability, cell apoptosis was determined by FACS. As shown in Fig. 3c, compared with the control groups, significant increases in cell apoptosis were observed in the HGC-27 OE and NCI-87 OE cells $(P<0.05)$.

Sphere forming ability is also a hallmark of CSCs $[20,23]$. Therefore, the sphere forming capacity of RNF43 OE cells and control cells from HGC-27 and N87 cell lines was measured by culturing cells with serum-free medium in ultralow-attachment 24-well plates. After 7 days in culture, tumorspheres with a diameter over $100 \mu \mathrm{m}$ were counted. The RNF43 OE group formed significantly fewer spheres than in the control groups (HGC27 control: $62 \pm 5.1$ spheres/well, OE: $28 \pm 4.3$ spheres/ well, $P<0.01$; N87 control: $51 \pm 5.8$ spheres/well, OE: $25 \pm 3.4$ spheres/well, $P<0.01$ ) (Fig. $4 \mathrm{a}$ ).
CD44 has been suggested to be the cell surface marker for gastric CSCs, but it lacks specificity due to inconsistent findings [4]. Recent studies have reported that combined CD44 and CD54 might be more accurate stem cell markers for GC [5]. To more closely determine the effect of RNF43 OE on GCSLCs, a flow cytometry assay was performed to detect CD44 and CD54 expression in RNF43 OE cells and control cells. The results showed that the percentage of CD44 and CD54 double-positive cells was lower in the RNF43 OE group compared with the control group (HGC-27, $6.3 \pm 0.6 \%$ vs $14.3 \pm 1.1 \%$, respectively, $P<0.05$; N-87, $1.8 \pm 0.2 \%$ vs $4.5 \pm 0.3 \%$, respectively, $P<0.05 \%$ ) (Fig. $4 \mathrm{c}$ ).

CSCs are considered to exhibit high tumorigenic abilities in xenografts, which reflects their in-vivo selfrenewal capability [24]. To gain insight into the role of RNF43 on tumorigenicity, equal numbers of SCs $\left(10^{4} /\right.$ group) formed by RNF43 OE N-87 cells (S87 OE) and $\mathrm{N}-87$ control cell lines (S87 Ctrl) were injected into two sides of rear flanks of NSG SCID mice (four mice per group) for subcutaneous xenografts. We then observed and measured the volume of subcutaneous tumors every week. The tumors derived from S87 OE mice grew much smaller and slower compared with the S87 Ctrl group $(P<0.01)$. Moreover, the proportion of $\mathrm{CD} 44^{+} \mathrm{CD} 54^{+}$cells in tumor cells digested from xenograft was measured using cytometry assay, and showed that S87 OE xenograft tumor possessed less $\mathrm{CD} 4^{+} \mathrm{CD} 54^{+}$cells compared with $\mathrm{S} 87$ Ctrl tumor, which was consistent with the findings in vitro, indicating the RNF43 OE remarkably suppresses $\mathrm{CD} 44^{+} \mathrm{CD} 54^{+}$cancer stem-like cells (Fig. 4b).

\section{RNF43 negatively regulates the Wnt/ $\beta$-catenin pathway}

The impact of RNF43 on cancer cells varies with cancer types [25]. To determine whether RNF43 regulates the Wnt signaling pathway in $\mathrm{GC}$, the key downstream proteins $\beta$-catenin, TCF-4, and $\mathrm{C}$-myc were measured using western blot analysis. Decreased expression of $\beta$-catenin, TCF4, and C-myc was observed in RNF43 OE cells compared with the Ctrl group (Fig. 5a). Moreover, RNF43 markedly downregulated the expression of Lgr5 protein, which is considered an upstream activator of the Wnt signaling pathway.

Because RNF43 affected the sphere forming ability of $\mathrm{OE}$ cells, we next examined the effect of addition of the Wnt signaling pathway ligand R-spondin 1 into sphere culture medium (Fig. 4a). After coculturing with R-spondin1, the OE cells regained their sphere forming capacity to some extent, but it was still less than in the control groups. Applying another Wnt pathway agonist (Wnt5a) also increased the expansion of tumorspheres from RNF43 OE cells. Conversely, blocking Wnt signaling by adding the Wnt antagonist 


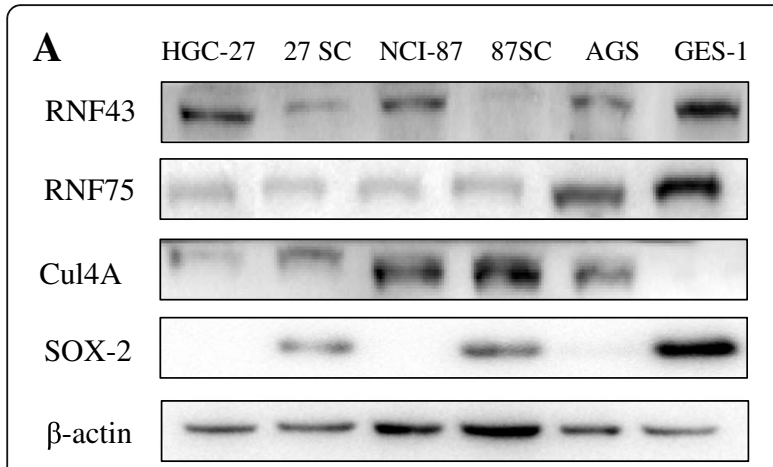

D

B

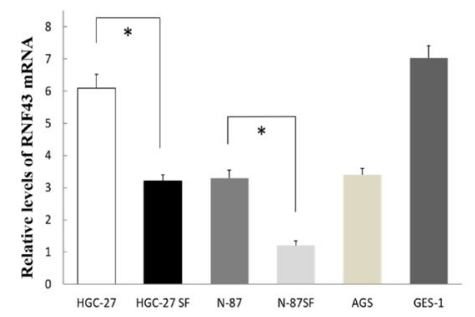

C

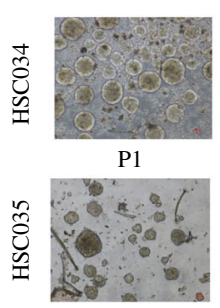

P1

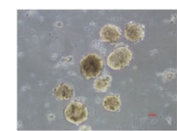

P2

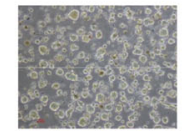

P2

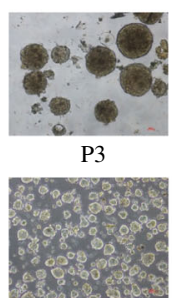

P3

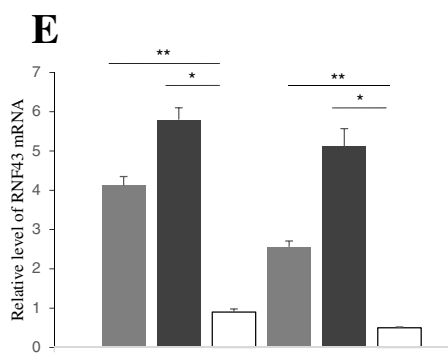

$\begin{array}{llllll}\mathrm{T} 1 & \mathrm{~N} 1 & \mathrm{SC} 1 & \mathrm{~T} 2 & \mathrm{~N} 2 & \mathrm{SC} 2\end{array}$

Fig. 2 Decreased expression of RNF43 protein in GC stem-like cells. a Western blot assay of RNF43, RNF75, Cul4a, and Sox-2 protein in cancer cells and HGC-27 and N87 GC stem-like cells. $\mathbf{b}$ qPCR of RNF43 mRNA expression in cancer cells and CG stem-like cells. Results represent mean \pm SD of three independent experiments. c Representative images of primary tumorsphere cells (upper, HSC034; bottom, HSC035). Most spheres were larger than $100 \mu \mathrm{m}$ in diameter after 7 days of culture. $P$ passage. $\mathbf{d}$ Western blot assay of RNF43 and Sox-2 protein in primary tumorsphere cells and corresponding cancer and adjacent normal tissues. e qPCR of RNF43 mRNA expression in primary tumorsphere cells and corresponding cancer and adjacent normal tissues. Results represent mean \pm SD of three independent experiments. ${ }^{*}$ means Statistically significant $(P<0.05) ;{ }^{* *}$ means Statistically significant $(P<0.01)$

DKK-1 in RNF43 OE cells yielded an opposing effect, leading to a reduced ability to from tumorspheres in vitro (Fig. 4a).

Previous in-vivo experiments revealed that RNF43 impaired tumorigenicity, and further immunohistochemical staining showed that RNF43 expression in xenograft tumor tissues had a negative correlation with $\beta$-catenin. In the RNF43 OE group, $\beta$-catenin was decreased and even lost in some regions, whereas in the control group $\beta$-catenin was relatively higher in tumor cells (Fig. 5b). In the control group, Sox-2 was expressed in part of the tissues, while in the RNF43 OE group Sox-2 was lost in the whole tissue.

\section{Discussion}

RNF43 is located on the cell surface, and competes with Lgr5 for binding with R-spondins [8]. The presence of Lgr5 or R-spondins affects RNF43 by ubiquitinating frizzled receptors and targeting them for degradation $[8,26]$. RNF43 also directly interacts with TCF4 and tethers TCF4 to the nuclear membrane, thus silencing TCF4 transcriptional activity [25].

RNF43 is mutated in several kinds of malignancies, including colorectal cancer, GC, and pancreatic cancer
$[25,26]$. The findings that RNF43 functions as an inhibitor against the Wnt signaling pathway are consistent with its mutation in cancers [27, 28]. However, in gastrointestinal malignancies, the results seemed to be paradoxical $[9,29]$. Our study confirmed that RNF43 expression was decreased in GC tissues compared with adjacent normal tissues. Moreover, RNF43 expression was significantly lower in SCs, which are considered a form of CSCs, than in ACs. A lack of association of other E3 ubiquitin ligases and stemness indicated a unique function of RNF43 in stem-like cancer cells. Our analyses also indicated that decreased RNF43 might be associated with TNM stage, metastasis, and intestinal type cancer, and survival analysis also implied its association with poor outcome of GC patients.

Our functional studies also showed that RNF43 inhibited cell growth and chemotherapy resistance in vitro, which are also regarded as hallmarks for CSCs. The chemotherapy-induced apoptosis in RNF43 OE cells might account for the impaired proliferation and drugresistance ability. Assays for other distinctive features for CSCs, such as tumorsphere forming ability in vitro and xenograft assays, also demonstrated that RNF43 OE would undermine self-renewal ability of GCSLCs. In 


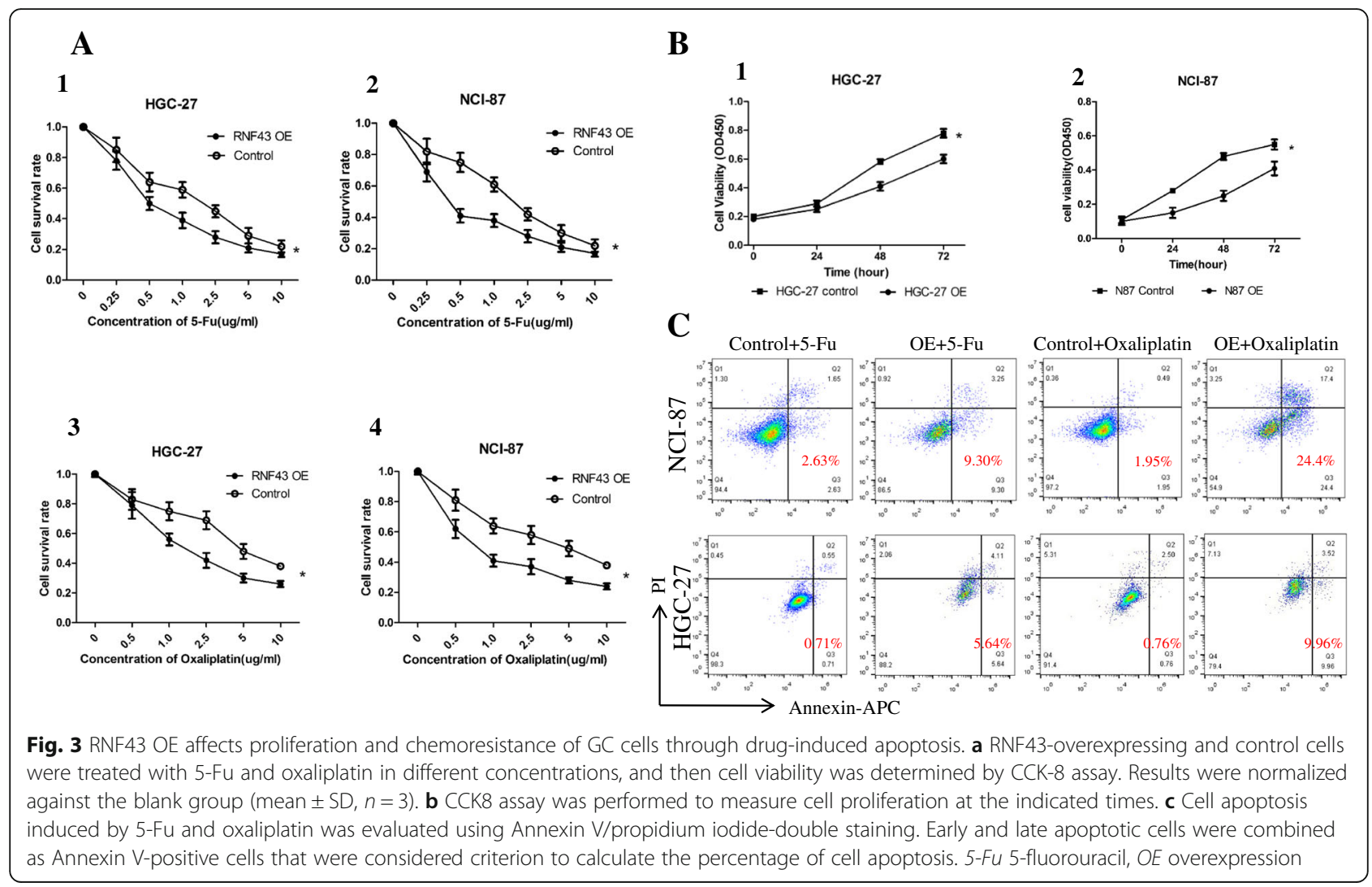

sphere culture, addition of the Wnt signaling pathway activators, R-spondin 1 and Wnt $5 \mathrm{a}$, could reverse this trend to some extent. Stem cell surface marker analysis could reveal cells with stem cell-like properties, and our results showed that stem cell markers were significantly decreased in the RNF43 OE group both in vitro and in vivo. In xenograft tumors, RNF43 OE was correlated with decreased expression of $\beta$-catenin and Sox-2, indicating that RNF43 would alter the Wnt/ $\beta$-catenin pathway and stemness in vivo.

Adenoviruses are small, single-strand DNA viruses that have been introduced in increasing clinical applications as a result of their stable gene delivery efficacy, high safety profile, and low pathogenicity [30]. Despite the growing number of studies on CSCs, there are still few therapies directly targeting CSCs [31]. Because the Ad-RNF43 we used in this study significantly inhibited viability of cells with low RNF43 expression compared with high RNF43 expression, our results might indicate a potential therapeutic application for Ad-RNF43 in GC CSC treatment.

The limitations of our study still need to be addressed. First, the SCs derived from primary GC tumors are too fragile to undergo virus infection, and thus the OE study was performed in established GC cell lines, which restrains the reliability of the conclusions. Second, surface marker analysis demonstrated that the stem-like cancer cells only account for a small population of the total cells, which might result in an inaccuracy of the effect of RNF43 on CSCs. This phenomenon might arise from the fact that CSCs could generate differentiated daughter cells [32]. Takaishi et al. [4] also found that CD44positive GC cells were able to generate CD44negative cells during culture, which retain selfrenewal capacity in just a small portion of cells. Third, due to the varied expression of RNF43 in different cancer cell lines, whether downregulation of RNF43 in highly expressed GC cell lines would render stemness to these cancer cells remains unclear, and needs more study to elucidate.

\section{Conclusion}

Our results showed that RNF43 is negatively correlated with GC patient clinical outcome and that the expression of RNF43 was significantly lower in GCSLCs than in ACs and normal mucosal cells. Furthermore, adenovirusinduced RNF43 OE inhibited tumor growth and stem cell-like phenotype through the canonical Wnt/ $\mathrm{W}$-catenin pathway both in vitro and in vivo. These findings suggest that the clinical value of adenovirus-mediated RNF43 targeting CSCs in GC is worth further exploration. 

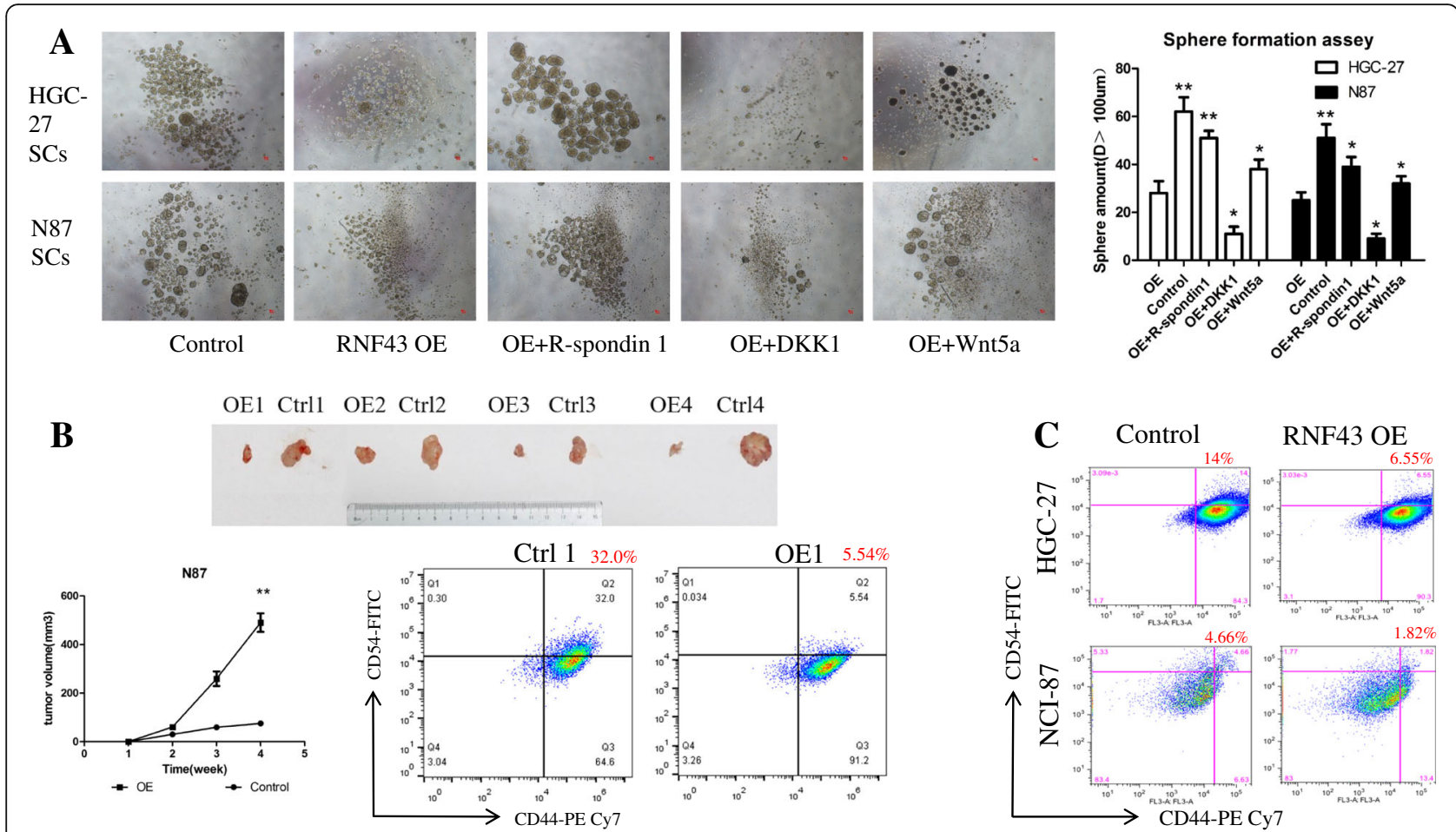

Fig. 4 RNF43 OE attenuates stem-like properties of GCSLCs. a Representative images (upper) of RNF43 OE cells that generated smaller and fewer tumorspheres compared with control groups. Tumorsphere count (right) in different treatment groups, which were consistent with the trend described. Data represent mean \pm SD of five independent experiments. ${ }^{*} P<0.05$. Scale Bar, $50 \mu \mathrm{m}$. $\mathbf{b}$ In-vivo assay of the effect of RNF43 on GC stem-like cell tumorigenesis. Upper: xenograft tumor driven by RNF43 OE and control GC cells. Lower left: during 30 days after tumor cell injection, RNF43 OE cells generate smaller tumors at a lower speed compared with the control group $(P<0.01)$. Lower right: representative flow cytometric analysis of candidate CSC markers CD44 and CD54 for GC stem-like cells from xenograft tumor formed by RNF43 overexpressing and control cells. c Representative flow cytometric analysis of CD44 and CD54 for GC stem-like cells formed by control and RNF43-overexpressing cells in HGC-27 and $\mathrm{NCl}-87$ cells. OE overexpression. ${ }^{* *}$ means Statistically significant $(P<0.01)$

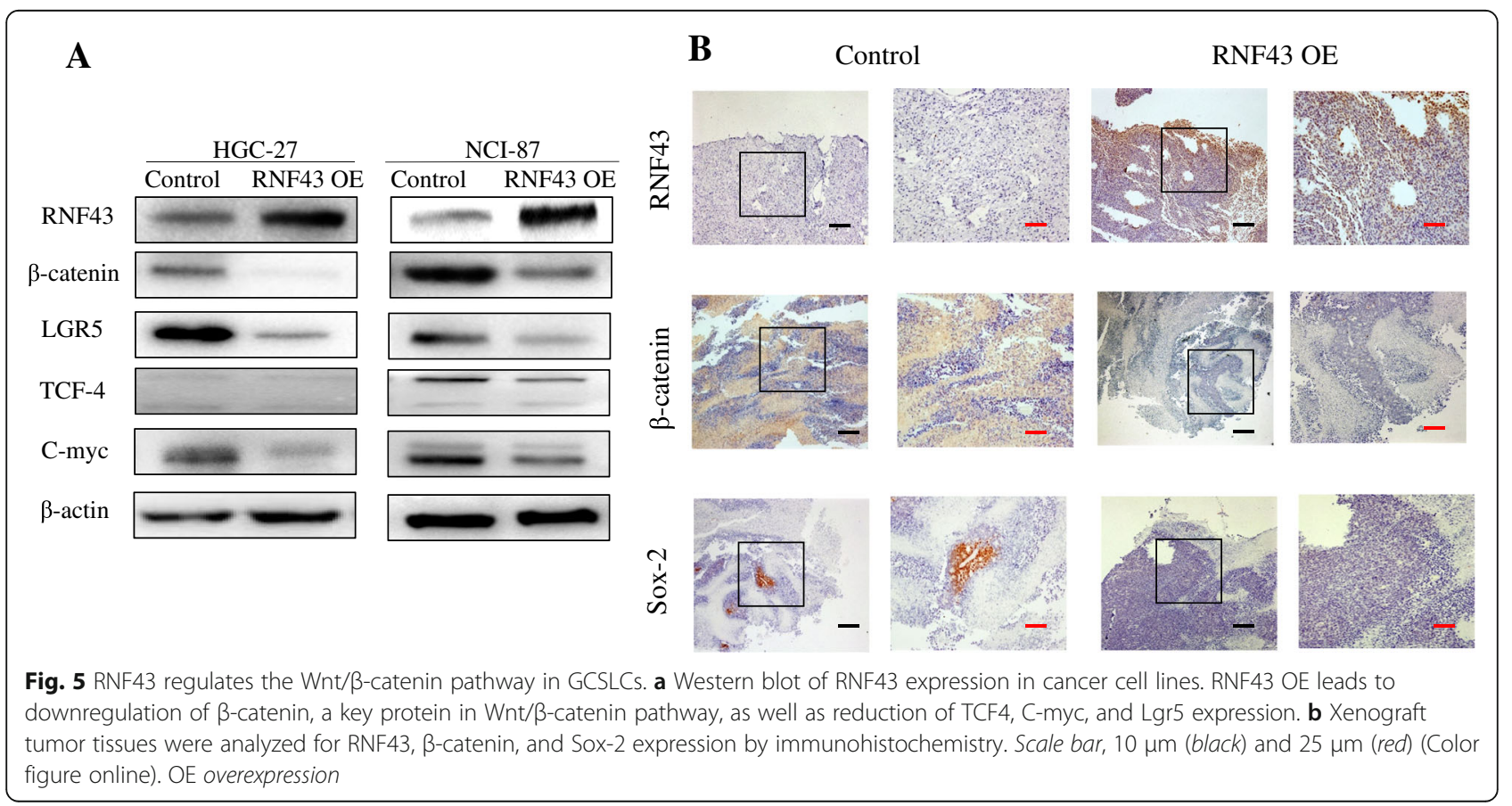




\section{Additional files}

Additional file 1: Figure S1. $I H C$ images of RNF43 in colon cancer, ovarian cancer, lung cancer, and their corresponding normal tissues (Scale bar, $10 \mu \mathrm{m}$ (black)). (PDF $187 \mathrm{~kb}$ )

Additional file 2: Figure S1. Gastric cancer cell lines in tumorsphere forming medium after 7 days (Scale bar, $50 \mu \mathrm{m}$ ). (PDF $186 \mathrm{~kb}$ )

Additional file 3: Figure S3. Infection efficiency of Ad-RNF43 in gastric cancer cells: left, observation under a regular microscope; right, observation of the same field under a fluorescence microscope (Scale Bar, $50 \mu \mathrm{m}$ ). (PDF $72 \mathrm{~kb}$ )

Additional file 4: Figure S4. CCK-8 assay of $\mathrm{HGC}-27$ and $\mathrm{NCl}-87$ treating with 5-Fu $(1 \mu \mathrm{g} / \mathrm{ml})$ and Oxaliplatin $(2.5 \mu \mathrm{g} / \mathrm{ml})$. (PDF $411 \mathrm{~kb})$

\section{Abbreviations}

AC: Adherent cell; bFGF: Basic fibroblast growth factor; CSC: Cancer stem cell; EGF: Epidermal growth factor; GC: Gastric cancer; GCSLC: Gastric cancer stem-like cell; IHC: Immunohistochemistry; Lgr5: Leucine-rich repeatcontaining G-protein-coupled receptor 5; OE: Overexpression; RNF43: Ring finger protein 43; SC: Sphere cell

\section{Acknowledgements}

The authors would like to thank Professor Wei Guo from Tsinghua University for suggestion in the study design.

\section{Funding}

This work was supported by grants from the National Program on Key Basic Research Project of China (No. 2014CBA02002), National Key Research and Development Plan (No. 2016YFC0905302), National Nature Science Foundation of China (No. 81272698, 81672319, 81602507), and Beijing Municipal Science and Technology Project (No. D131100005313010).

\section{Availability of data and materials}

Supporting data can be obtained from the first author.

\section{Authors' contributions}

$Y G, A C$, and LC conceived the project. HQX and $K Z$ collected the clinical samples. YG and JL performed immunohistochemical staining experiments. HQX and JC evaluated the $I H C$ slides. YG and XW performed $\mathrm{QPCR}$ and western blot analysis. $W X$ and $Y Z$ performed molecular biology experiments. $Y G$ and $J$ performed xenograft experiments, flow cytometry, and chemoresistance assay. YG, AC, and LC wrote the manuscript. BW collected and analyzed the clinical data. All authors read and approved the final manuscript.

\section{Competing interests}

The authors declare that they have no competing interests.

\section{Consent for publication}

All participants give their permission to publish this study.

\section{Ethics approval}

This study was approved by the Institutional Review Board of the Chinese PLA General Hospital (S2013-116-01) and all patients provided written informed consent. The animal model study was approved by the Tsinghua University Institutional Animal Care and Use Committee (IACUC) (Reference number: 2010-GW-A1), and were performed in compliance with the Animal Welfare Act and the Guide for the care and Use of Laboratory Animals.

\section{Publisher's Note}

Springer Nature remains neutral with regard to jurisdictional claims in published maps and institutional affiliations.

\section{Author details}

'Department of General Surgery, Chinese PLA General Hospital, Beijing 100853, China. ${ }^{2}$ School of Medicine, Tsinghua University, Beijing 10084, China
Received: 12 November 2016 Revised: 23 February 2017

Accepted: 31 March 2017 Published online: 26 April 2017

\section{References}

1. Stewart BW. World Cancer Report 2014. Lyon: International Agency for Research on Cancer; 2015. http://publications.iarc.fr/Non-Series-Publications/ World-Cancer-Reports/World-Cancer-Report-2014. Accessed 1 Sept 2015.

2. Hartgrink HH, Jansen EP, van Grieken NC, van de Velde CJ. Gastric cancer. Lancet. 2009:374(9688):477-90.

3. Clarke MF, Dick JE, Dirks PB, Eaves CJ, Jamieson CH, et al. Cancer stem cells-perspectives on current status and future directions: AACR Workshop on cancer stem cells. Cancer Res. 2006;66(19):9339-44.

4. Takaishi S, Okumura T, Tu S, Wang SS, Shibata W, et al. Identification of gastric cancer stem cells using the cell surface marker CD44. Stem Cells. 2009:27(5):1006-20.

5. Chen T, Yang K, Yu J, Meng W, Yuan D, et al. Identification and expansion of cancer stem cells in tumor tissues and peripheral blood derived from gastric adenocarcinoma patients. Cell Res. 2012;22(1):248-58.

6. Zhang $X$, Hua $R$, Wang $X$, Huang M, Gan L, et al. Identification of stem-like cells and clinical significance of candidate stem cell markers in gastric cancer. Oncotarget. 2016;7(9):9815-31.

7. Rassouli FB, Matin MM, Saeinasab M. Cancer stem cells in human digestive tract malignancies. Tumour Biol. 2016;37(1):7-21.

8. Koo BK, Spit M, Jordens I, Low TY, Stange DE, et al. Tumour suppressor RNF43 is a stem-cell E3 ligase that induces endocytosis of Wnt receptors. Nature. 2012:488(7413):665-9.

9. Yagyu R, Furukawa Y, Lin YM, Shimokawa T, Yamamura T, Nakamura Y. A novel oncoprotein RNF43 functions in an autocrine manner in colorectal cancer. Int J Oncol. 2004;25(5):1343-8.

10. Wu J, Jiao Y, Dal Molin M, Maitra A, De Wilde RF, et al. Whole-exome sequencing of neoplastic cysts of the pancreas reveals recurrent mutations in components of ubiquitin-dependent pathways. Proc Natl Acad Sci U S A 2011;108(52):21188-93.

11. Ryland GL, Hunter SM, Doyle MA, Rowley SM, Christie M, et al. RNF43 is a tumour suppressor gene mutated in mucinous tumours of the ovary. J Pathol. 2013;229(3):469-76

12. Ong CK, Subimerb C, Pairojkul C, Wongkham S, Cutcutache I, et al. Exome sequencing of liver fluke-associated cholangiocarcinoma. Nat Genet. 2012;44(6):690-3.

13. Cancer Genome Atlas Research Network. Comprehensive molecular characterization of gastric adenocarcinoma. Nature. 2014;513(7517):202-9.

14. Wang K, Yuen ST, Xu J, Lee SP, Yan HH, et al. Whole-genome sequencing and comprehensive molecular profiling identify new driver mutations in gastric cancer. Nat Genet. 2014:46(6):573-82.

15. Zebisch M, Xu Y, Krastev C, MacDonald BT, Chen M, et al. Structural and molecular basis of ZNRF3/RNF43 transmembrane ubiquitin ligase inhibition by the Wnt agonist R-spondin. Nat Commun. 2013;4:2787.

16. Xi HQ, Cai AZ, Wu XS, Cui JX, Shen WS, et al. Leucine-rich repeat-containing G-protein-coupled receptor 5 is associated with invasion, metastasis, and could be a potential therapeutic target in human gastric cancer. $\mathrm{Br} J$ Cancer. 2014;110(8):2011-20

17. Li XB, Yang G, Zhu L, Tang YL, Zhang C, et al. Gastric Lgr5(+) stem cells are the cellular origin of invasive intestinal-type gastric cancer in mice. Cell Res. 2016:26(7):838-49.

18. Barker $N$, Huch $M$, Kujala $P$, van de Wetering $M$, Snippert HJ, et al. Lgr5(+ve) stem cells drive self-renewal in the stomach and build long-lived gastric units in vitro. Cell Stem Cell. 2010;6(1):25-36.

19. Barker N, van Es JH, Kuipers J, Kujala P, van den Born M, et al. Identification of stem cells in small intestine and colon by marker gene Lgr5. Nature. 2007:449(7165):1003-7.

20. Vescovi AL, Galli R, Reynolds BA. Brain tumour stem cells. Nat Rev Cancer. 2006:6(6):425-36.

21. Vargo-Gogola T, Rosen JM. Modelling breast cancer: one size does not fit all. Nat Rev Cancer. 2007:7(9):659-72.

22. Yu D, Shin HS, Choi G, Lee YC. Proteomic analysis of CD44(+) and CD44(-) gastric cancer cells. Mol Cell Biochem. 2014;396(1-2):213-20.

23. Song Z, Yue W, Wei B, Wang N, Li T, et al. Sonic hedgehog pathway is essential for maintenance of cancer stem-like cells in human gastric cancer. PLoS One. 2011;6(3):e17687. doi:10.1371/journal.pone.0017687.

24. Kreso A, Dick JE. Evolution of the cancer stem cell model. Cell Stem Cell. 2014;14(3):275-91. 
25. Loregger A, Grandl M, Mejías-Luque R, Allgäuer M, Degenhart K, et al. The E3 ligase RNF43 inhibits Wnt signaling downstream of mutated $\beta$-catenin by sequestering TCF4 to the nuclear membrane. Sci Signal. 2015;8(393):ra90.

26. Jiang $X$, Charlat O, Zamponi R, Yang Y, Cong F. Dishevelled promotes Wnt receptor degradation through recruitment of ZNRF3/RNF43 E3 ubiquitin ligases. Mol Cell. 2015;58(3):522-33.

27. Yan HH, Lai JC, Ho SL, Leung WK, Law WL, et al. RNF43 germline and somatic mutation in serrated neoplasia pathway and its association with BRAF mutation. Gut. 2016. doi:10.1136/gutjnl-2016-311849.

28. Min BH, Hwang J, Kim NK, Park G, Kang SY, et al. Dysregulated Wnt signalling and recurrent mutations of the tumour suppressor RNF43 in early gastric carcinogenesis. J Pathol. 2016; 240(3):304-14. doi:10.1002/path.4777.

29. Wang G, Fu Y, Yang X, Luo X, Wang J, et al. Brg-1 targeting of novel miR550a-5p/RNF43/Wnt signaling axis regulates colorectal cancer metastasis. Oncogene. 2016:35(5):651-61.

30. Kotterman MA, Schaffer DV. Engineering adeno-associated viruses for clinical gene therapy. Nat Rev Genet. 2014;15(7):445-51.

31. Taniguchi H, Moriya C, Igarashi H, Saitoh A, Yamamoto H, et al. Cancer stem cells in human gastrointestinal cancer. Cancer Sci. 2016;107(1):1556-62. doi: 10.1111/cas.13069

32. Sato R, Semba T, Saya H, Arima Y. Concise Review: Stem cells and epithelialmesenchymal transition in cancer: biological implications and therapeutic targets. Stem Cells. 2016;34(8):1997-2007.

\section{Submit your next manuscript to BioMed Central} and we will help you at every step:

- We accept pre-submission inquiries

- Our selector tool helps you to find the most relevant journal

- We provide round the clock customer support

- Convenient online submission

- Thorough peer review

- Inclusion in PubMed and all major indexing services

- Maximum visibility for your research

Submit your manuscript at www.biomedcentral.com/submit 